\title{
Health Care Utilization
}

Faculty Discussant: A. Simon Pickard, PhD

Article 1: Real-World Cost-Effectiveness Analysis of Allogeneic Hematopoietic Stem Cells

Transplantation vs. Hydroxyurea Therapy in Children with Sickle Cell Disease Jifang Zhou, MD, MPH; Gregory S. Calip, PharmD, MPH, PhD

Article 2: Fondaparinux Sodium Compared with Low Molecular Weight Heparins for Thromboprophylaxis among the Patients with Orthopedic Immobility: A Systematic Review and Meta-Analysis

Arun Kumar PharmD, MS; Ashna Talwar, BPharm; Wenchen Wu, PhD; Joel F Farley, PhD

Article 3: Differences by Primary Insurance in 30-day All-cause Readmission for Patients Undergoing Breast Cancer Surgeries

Ming Chen, MS, MSPharm; Shelley Irene White-Means, PhD, MA; Sam Li, PhD, MS

Article 4: Seasonal Variation in Heart Failure Hospitalizations in the United States

Kendra Y. Foster, MS; Linnea A. Polgreen, PhD

Article 5: Antidiabetic Medications among People 20 years and Older and with Diabetes in the United States

Julie Zhiying Zhao, MPH; Angeline Carlson, PhD

Article 6: Healthcare Utilization in Type 2 Diabetes Patients Using DPP-4 inhibitors and GLP-1

Agonists

Sajan Pandya BPharm; Monica Hwang, PhD

Article 7: Estimating the Impact of the Food and Drug Administration's (FDA) Unapproved Drug Initiative (UDI) on Drug Prices and Expenditures

Sharma D; Schumock GT; Saffore C; Edwards SA; Walton SM 
Health Care Utilization Faculty Discussant: A. Simon Pickard, PhD

Professor and Director of Graduate Studies, Department of Pharmacy Systems, Outcomes and Policy, University of Illinois at Chicago

833 S. Wood Street, Room 254, MC 871, Chicago, IL 60612

Email: pickard1@uic.edu

The themes in this session on health care utilization explored issues related to the cost of health care and prescription drug use. These studies are typically conducted using secondary data sources, such as claims data or population survey data. Foster and Polgreen applied time series analysis to examine seasonal variation in heart failure, while Zhao and Carlson reported trends in self-reported blood glucose control and use of antihyperglycemics. The Nationwide Re-admission Database (NRD) was used by Chen et al to determine whether readmission rates differed by insurance status among patients who underwent breast cancer surgery. Zhou and Calip examined the cost of an innovative therapy by comparing allogenic hematiopoietic stem cells transplantation versus hydroxyurea therapy in children with sickle cell disease. In addition to examining patterns of medication use and comparing the costs or effectiveness of treatments, secondary data also can be used to evaluate the impact of pharmaceutical policy. This was the focus of the investigation by Sharma and colleagues of the FDA's unapproved drug initiative (UDI) on drug prices and expenditures which generated some remarkable results on the subsequent prices of generic drugs. In the spirit of the meeting, proposed studies yet to be conducted were also presented, including Sajan Pandya's examining of patients initiating therapies with DPP-4 inhibitors and GLP-1 agonists using the Medical Expeditures Panel Survey (MEPS). Alternatively, systematic reviews of the literature can be used to conduct studies such as meta-analyses to compare the effectiveness of treatment, as was done by Kumar et al in their comparison of fondaparinux to low molecular weight heparins for thrombopropylaxis. The presentations in this session demonstrated the ongoing importance using established methodologies and secondary data to understand patterns of use of medications and to compare the clinical and economic outcomes drug treatments to better inform medical decision making. It also highlighted the need for additional information to inform patient centered care, such as patient preferences for treatment, that should supplement information about clinical outcomes and costs of treatment, particularly when there are tradeoffs to be made between clinical and economic outcomes. 


\section{Real-World Cost-Effectiveness Analysis of Allogeneic Hematopoietic Stem Cells Transplantation vs. Hydroxyurea Therapy in Children with Sickle Cell Disease}

Jifang Zhou, MD, MPH

Graduate Student

University of Illinois at Chicago

Center for Pharmacoepidemiology and Pharmacoeconomic Research

833 S. Wood St. MC 871, Chicago, IL 60612

izhou86@uic.edu

Gregory S. Calip, Pharm.D. M.P.H., Ph.D.

Assistant Professor

University of Illinois at Chicago

Center for Pharmacoepidemiology and Pharmacoeconomic Research

833 S. Wood St. MC 871, Chicago, IL 60612

gcalip@uic.edu

Acknowledgements: This study was funded by the UIC/AbbVie HEOR Fellowship.

\section{ABSTRACT}

Objective: To compare the real-world cost-effectiveness of hematopoietic stem cells transplantation (HSCT) and Hydroxyurea $(H U)$ in a commercially-insured U.S. population of sickle cell disease (SCD) patients aged $\leq 17$ years. The primary outcomes were all-cause per person per month costs and all-cause acute care encounters defined as inpatient admissions and emergency room (ER) visits.

Methods: We conducted a population-based cohort study using the Truven Health MarketScan ${ }^{\circledR}$ Research databases (20092014). Pediatric patients with SCD undergoing allogeneic HSCT and those newly initiating HU (index date: stem cell infusion and prescription dispensing, respectively) with continuous health plan enrollment for $\geq 6$ months before and after the index date were included.

Results: We identified 16 HSCT recipients and 400 pediatric HU initiators. The mean (SD) age at cohort entry were 8.8 (4.6) and 10.0 (4.6) years among pediatric and adolescent patients with SCD. Prior to the index date, HSCT recipients had greater occurrence of iron overload (37.5\% vs. $7.8 \% ; p<0.0001)$ and stroke (12.5\% vs. $2.5 \% ; p=0.0191)$. Over a median follow-up of 27 and 22 months post-transplantation and HU initiation, the mean (SD) all-cause per person per month (PPPM) acute care utilization were similar (0.21 (0.36) vs. $0.21(0.26), p=0.9641)$. HSCT recipients had significantly lower mean (SD) PPPM SCDrelated acute care utilization $(0.02(0.03)$ vs. $0.12(0.19), p<0.0001)$; despite higher mean (SD) PPPM health costs $(\$ 19,062$ $(14,600)$ vs. $\$ 2,485(3,492)$, in 2014 U.S. dollars; $p=0.0004)$. Only modest differences in all-cause health expenditures persisted beyond the first year post-treatment (mean (SD), $\$ 3,593(5,565)$ vs. $\$ 2,783(5,031) ; p=0.5590)$.

Conclusion: We found lower SCD-related acute care utilization following HSCT and similar all-cause health expenditures relative to $\mathrm{HU}$ therapy following one year post-transplantation among pediatric patients with SCD.

Key words: Cost-effectiveness analysis, Sickle cell disease, Hematopoietic stem cells transplantation, Hydroxyurea 


\title{
Fondaparinux Sodium Compared with Low Molecular Weight Heparins for Thromboprophylaxis among the Patients with Orthopedic Immobility: A Systematic Review and Meta-Analysis
}

Arun Kumar PharmD, MS

Graduate Student

University of Minnesota,

College of Pharmacy

308, Harvard Street SE, Minneapolis, MN 55455

Kumar468@umn.edu

Ashna Talwar, BPharm

Graduate Student

St. John's University, Queens, NY

College of Pharmacy and Health Sciences,

8000, Utopia Parkway, Jamaica, NY-11439

ashna.talwar16@my.stjohns.edu

\author{
Wenchen Wu, PhD \\ Associate Professor, \\ St. John's University, Queens, NY \\ College of Pharmacy and Health Sciences, \\ 8000, Utopia Parkway, Jamaica, NY-11439 \\ wuw@stjohns.edu \\ Joel F Farley, PhD \\ Professor, \\ University of Minnesota, \\ College of Pharmacy \\ 308, Harvard Street SE, Minneapolis, MN 55455 \\ farl0032@umn.edu
}

\begin{abstract}
Objectives: The objective of this meta-analysis was to systematically review the randomized clinical trials (RCTs) of Fondaparinux Sodium once daily versus low molecular weight heparins (LMWH) at the titrated dose for the prophylaxis of venous thromboembolism (VTE) among the patients with orthopedic immobility.

Methods: Systematic search in the database e.g. EMBASE, MEDLINE, Cochrane Library, ProQuest, Science Direct, Google Scholar and clinicaltrials.gov was done to identify RCTs evaluating both treatments for the prophylaxis of VTE published in English language from the year 2000 to 2017. A web-based systematic review tool "Covidence" was used to systematically screen the studies of interest. Analysis in "RevMan" was performed with the relative odds based on the fixed and random effect models. Results were presented as odds ratios (OR) with their $95 \%$ confidence intervals. The assessment of study quality and risk of bias among the included studies was performed as per Cochrane collaboration.
\end{abstract}

Results: After screening 10,644 articles, 4,721 were selected for review. Nine RCTs were included in the final analysis. Pooled analyses showed a significant $58 \%$ reduction for VTE $(O R=0.42[0.32,0.56])$ and $5 \%$ reduction in mortality $(O R=0.95$ $[0.64,1.40])$ with Fondaparinux Sodium compared to LMWH. Contrarily, there was a significant 55\% increase in the risk for major bleeding $(\mathrm{OR}=1.55[1.15,2.10])$ and $15 \%$ increase in minor bleeding (OR=1.16 $[0.90,1.50])$ with Fondaparinux Sodium compared to LMWH. Quality assessment results identified three of the nine studies at high risk for bias due to "Blinding of Participants and Personnel".

Conclusions: This meta-analysis concluded that Fondaparinux Sodium was superior to LMWH in reduction of VTE and mortality but increased the risk for major bleeding. Pharmacists should be aware of potential adverse effects from administering Fondaparinux Sodium with orthopedic immobility.

Keywords: Fondaparinux Sodium. Low Molecular Weight Heparins, Venous thromboembolismKey words: Costeffectiveness analysis, Sickle cell disease, Hematopoietic stem cells transplantation, Hydroxyurea 


\section{Differences by Primary Insurance in 30-day All-cause Readmission for Patients Undergoing Breast Cancer Surgeries}

Ming Chen, MS, MSPharm*

Research Assistant

University of Tennessee Health Science Center

College of Pharmacy

881 Madison Avenue, Memphis, TN 38163

mchen46@uthsc.edu

Shelley Irene White-Means, PhD, MA

Professor

University of Tennessee Health Science Center

College of Pharmacy

881 Madison Avenue, Memphis, TN 38163

swhiteme@uthsc.edu
Sam Li, PhD, MS

Assistant Professor

University of Tennessee Health Science Center

College of Pharmacy

881 Madison Avenue, Memphis, TN 38163

mli54@uthsc.edu

*Conference Presenter and Reprints: Ming Chen, MS, MSPharm, Univeristy of Tennessee Health Science Center, Memphis, TN 38103 (email: mchen46@uthsc.edu).

This study was self-funded. The University of Tennessee Health Science Center exempted this study from approval of the institutional review board (IRB). All authors have no conflict of interest.

\section{ABSTRACT}

Introduction: Previous studies have found racial and socio-economic disparities in breast cancer hospitalization outcomes. However, whether readmission outcomes following the hospitalization differ by primary insurance is largely unknown.

Objective: To examine whether 30-day all-cause readmissions differ by primary insurance among patients who underwent breast cancer surgeries.

Methods: A retrospective observational study using Nationwide Readmission Database (NRD) were conducted to identify patients (aged $\geq 40$ yrs) undergoing breast cancer surgeries in 2015. Our outcome of interest was 30-day risk-standardized all-cause readmission. Outcomes were estimated and stratified by primary insurance. Hierarchical logistic regression models accounting for hospital clustering were performed to examine predictors of readmission. Additional analysis using risk adjustment and reliability adjustment were conducted to assess hospital variability in readmission metric.

Results: Of 5,150 patients undergoing breast cancer surgeries at 1,103 hospital sites, 277 (5.4\%) patients were reamitted within 30 days. Diabetes [aOR: 1.74 (1.22-2.50)], COPD [aOR: 1.47 (1.02-2.13)] and higher risk of mortality [aOR: 1.69 (1.132.51) moderate vs mild; 3.87(2.16-6.93) major \& extreme vs mild] were significant predictors for readmissions. In subgroup analysis, an increased risk of readmission was associated with large hospital bedsize in the private-insured group [aOR: 2.11 (1.01-4.42) vs small], however, none of the hospital characteristics had a significant effect on readmissions in the Medicare \& Medicaid group. Risk-adjusted readmission rates varied widely across overall hospitals [ICC: 10.63\%]; wider variation existed in subgroup analysis [ICC: $17.97 \%$ (Medicare \& Medicaid) vs $21.11 \%$ (private insurance)]. Mean risk-standardized hospital-wide readmission rates [Mean: 6.22 (SD: 2.01)] were higher for Medicare \& Medicaid patients compared to the private-insured [Mean: 4.86 (SD: 1.43)]. Of 523 hospitals having patients with both insurance, 465 (88.9\%) hospitals presented higher readmission rates for Medicare \& Medicaid patients, that were more likely to be large (50.8\%), private invest-owned (16.3\%), teaching (55.1\%) hospitals with large operative volume $(82.4 \%)$ of the breast cancer surgeries.

Conclusion: Using a national sample of hospitalized patients with breast cancer surgeries, differences in readmission metrics by primary insurance existed. Lower hospital readmission rates but higher variation was observed among private-insured patients compared to Medicare \& Medicaid patients. These findings suggest that higher readmission rates may be a quality metric for lower hospital care where hospitalized private-insured patients received the treatments following the breast cancer surgeries.

*** Abbreviation: aOR $=$ adjusted odds ratio; ICC = intracluster correlation coefficient $* * *$

Keywords: breast cancer surgeries; readmission; insurance; risk adjustment; quality of care 


\section{Seasonal Variation in Heart Failure Hospitalizations in the United States}

Kendra Y. Foster, MS

Graduate Student

University of lowa

College of Pharmacy

115 S. Grand Avenue, lowa City, lowa 52242

Kendra-foster@uiowa.edu

Linnea A. Polgreen, PhD

Professor

University of lowa

College of Pharmacy

115 S. Grand Avenue, lowa City, lowa 52242

linnea-polgreen@uiowa.edu

\section{ABSTRACT}

Introduction: Heart Failure (HF) is a significant cause of morbidity and mortality in the United States. One of the main risk factors for $\mathrm{HF}$ is a previous acute myocardial infarction (AMI). It has been shown that AMIs are seasonal with higher rates occurring in winter months. Since there is a seasonal pattern to AMIs, there was interest in determining if $\mathrm{HF}$ hospitalization rates may also have a seasonal pattern.

Methods: Using the National Inpatient Sample database during the years 1998-2011, we identified all hospitalizations with primary CCS codes for $\mathrm{HF}$ for patients who were 18 years of age or older. To estimate monthly incidence, we aggregated by year and month and applied appropriate weights. We fit a collection of time series models to determine the trend and seasonality of $\mathrm{HF}$ hospitalizations to determine how it varies across regions within the US.

Results: There were $13,284,216$ hospitalizations due to HF during the study period. Our model suggests that the risk for hospitalization due to $\mathrm{HF}$ is seasonal with the peak months being winter months. The highest risk month was February, while the lowest risk month was August. The seasonality was consistent across the census bureau-designated regions.

Conclusion: As we expected, HF hospitalizations are seasonal with the peak months being during winter. These results indicate the importance of management of HF. The specific reason that HF exhibits a seasonal pattern is unknown.

Therefore, more information regarding environmental risk factors may lead to better prevention of hospitalizations, which can significantly reduce $\mathrm{HF}$ cost in the United States.

Key Words: Heart Failure, Seasonality, Time Series, Trends 


\section{Antidiabetic Medications among People 20 years and Older and with Diabetes in the United States}

Julie Zhiying Zhao, MPH

Graduate Student

College of Pharmacy

University of Minnesota

zhaox086@umn.edu

Angeline Carlson, PhD

Adjunct Professor

College of Pharmacy

University of Minnesota

angie@dataiq.com

\section{ABSTRACT}

Introduction: Among people 18 years or older the prevalence of diabetes in the United States in 2015 was approximately 30.2 million people, or $12.2 \%$. This study examines the association between glycohemoglobin (HbA1c) levels and reported use of antidiabetic medications for persons with a diagnosis of diabetes.

Methods: The 2013-2014 National Health and Nutrition Examination Survey (NHANES) was used for analysis. Persons were identified based on response to a health survey question regarding diagnosis of diabetes by a health care professional. $\mathrm{HbA} \mathrm{CC}$ values were provided in the lab data file; antidiabetic medications were identified using generic drug name available in the prescription medication data file. Chi square test compared differences in antidiabetic medication utilization by $\mathrm{HbA} 1 \mathrm{c}$ level group (<=7\% vs $>7 \%$ ). P value $<0.05$ was considered statistically significant.

Results: Among US adults (>=20 years old) there were 707 who reported a diagnosis of diabetes and included in analysis. This corresponds to a weighted prevalence of $10.1 \%$ (95\% Cl: 9.0-11.1). About 48\% were aged $45-64$ years old; $51 \%$ were female. The proportion with $\mathrm{HbA} 1 \mathrm{c}>7 \%$ was $46 \%$. Overall, $83.3 \%$ reported use of antidiabetic medications; $13.5 \%$ with both insulin and oral antidiabetic agents, $14.8 \%$ insulin only; $55 \%$ oral antidiabetic agents only. The proportion of patients reporting any use of insulin was significantly higher among persons with $\mathrm{HbA} 1 \mathrm{c}>7 \%$ than among those with $\mathrm{HbA} 1 \mathrm{c}<=7 \%$ (45\% vs $15 \%$, respectively; $\mathrm{p}<.0001)$. Reported use of oral antidiabetic agents only was significantly higher among patients with $\mathrm{HbA1 \textrm {c }}$ $<=7 \%$ (62\% vs 47\%; $<$ <.0001). Among persons using oral antidiabetic medications, use of sulfonylureas was significantly higher among patients with $\mathrm{HbA} 1 \mathrm{c}>7 \%(33 \%$ vs $18 \%$; $\mathrm{p}<.0001)$.

Conclusions: About $46 \%$ of US adults reporting diabetes did not achieve an HbAlc target suggesting glycemic control. Healthcare professionals might identify more effective treatments or diabetes care managements to control glucose level in these patients. 


\section{Healthcare Utilization in Type 2 Diabetes Patients Using DPP-4 inhibitors and GLP-1 Agonists}

Sajan Pandya BPharm

Email: sajan.pandya17@my.stjohns.edu

Cell Phone: (917) 495-6488

Monica Hwang, PhD

St. John's University, Queens, NY

Office Phone: (718) 990-5936

\section{Acknowledgements: N/A}

\section{ABSTRACT}

Introduction. According to Center for Disease Control, 30.3 million people (approximately 9.4\%) of the US population has diabetes with prevalence only set to increase. Metformin is the preferred pharmacologic choice in the management of type 2 diabetes mellitus (T2DM). However, if glucose level is uncontrolled with metformin, a second agent is added. The choice for the second agent includes dipeptidyl peptidase-4 (DPP-4) inhibitors and glucagon-like peptide-1 (GLP-1) agonists. Although there have been studies associated with clinical outcomes of DPP-4 inhibitors and GLP-1 agonists, there have been limited studies describing healthcare utilization associated with initiating these therapies.

Objectives: The objective of this study is to examine healthcare utilization of T2DM patients initiating therapies with DPP-4 inhibitors and GLP-1 agonists using the Medical Expenditure Panel Survey (MEPS).

Theoretical Framework: Andersen healthcare utilization model will be used in this study. According to the model, use of health services is determined by: predisposing factors, enabling factors, and need. In this study, predisposing factors such as age and race and enabling factors such as access to and type of health insurance will be examined.

Proposed Methods: This study will use MEPS panel 19 longitudinal data file and household component events file from 2014 and 2015. Patients with T2DM will be identified from medical conditions file using ICD-9 code of 250. Patients on DPP-4 inhibitors and GLP-1 agonists will be identified from prescribed medicines file. Then to filter patients initiating therapy with DPP-4 inhibitors or GLP-1 agonists, variables RXBEGMM and RXBEGYRX will be used. All patients initiating therapy between January 1, 2014 to December 31, 2015 and continuing therapy for at least 6 months will be included in the study. Healthcare utilization for six months after initiating therapy will be examined.

Key Words: Healthcare utilization, Type 2 diabetes, DPP-4 inhibitors, GLP-1 agonists, MEPS 


\section{Estimating the Impact of the Food and Drug Administration's (FDA) Unapproved Drug Initiative (UDI) on Drug Prices and Expenditures}

Sharma D, Schumock GT, Saffore C, Edwards SA, Walton SM

Department of Pharmacy Systems, Outcomes and Policy, College of Pharmacy

University of Illinois at Chicago, Chicago, IL

\section{ABSTRACT}

Introduction: The 2006 FDA-UDI was implemented to help remove unsafe/ ineffective drugs. Manufacturers of such drugs could conduct studies to prove safety and/or efficacy and file a New Drug Application, for which approval provided limited market exclusivity. To date, very few studies have examined consequences of this policy on post-approval prices and quantities sold.

Objective: Measure the impact on prices, expenditures, quantities sold, and price elasticities for drugs approved under the FDA-UDI.

Methods: The DESI-II list prepared by the FDA Prescription Drug Wrap-Up program of 1984 was systematically searched to identify single-entity unapproved marketed prescription drugs. For the drugs which obtained voluntary FDA approval within the FDA-UDI from 2006-2015, a retrospective analysis was conducted using IQVIA National Sales Perspective database to analyze trends in total expenditures, units sold, price/unit and price elasticities. All prices were adjusted to 2017 dollars.

Results: Seventeen previously-marketed unapproved drug products were identified and 12 were included in the analysis (five excluded due to missing data). Compared to two years before approval, a steep increase in price and expenditures was observed two years post-approval for all except one with mean and median absolute percentage change of 1,684\% (SD: $3,358 \%$ ) and $275 \%$ (Range: $+10,729 \%$ to $-46 \%$ ) for price and 1,167\% (SD: $2.072 \%$ ) and $343 \%$ (Range: $-14 \%$ to $6,679 \%$ ) for expenditure. Substantial variance was observed in the changes in units sold. The demand for all except two products was found to be price inelastic.

Conclusion and Implication: A marked increase was seen in post-approval prices and expenditures for the vast majority of drugs approved in the FDA-UDI with highly variable corresponding changes in units sold. As the FDA-UDI is still ongoing, consideration of the impact on post-approval drug prices should be given along with added assurances of safety and efficacy of these drug products and reform should be considered. 